Anuario Latinoamericano Ciencias Políticas

y Relaciones Internacionales

vol. 8, 2019

pp. $87-100$

\section{Los dilemas de la participación política de las mujeres en México. Retos y retrocesos ante el avance sustantivo de las mujeres}

DOI: $10.17951 /$ al.2019.8.87-100

\section{The Dilemmas of Women's Political Participation in Mexico. Challenges and Setbacks to the Substantive Progress of Women}

\author{
Mónica Rosado Toledo* \\ ESCUELA NACIONAL DE ANTROPOLOGÍA E HISTORIA \\ CIUDAD DE MÉXICO, MÉXICO \\ $\triangle$ moni.r.toledo@gmail.com \\ https://orcid.org/0000-0001-9651-0838 \\ Lizeth Pérez Cárdenas** \\ UNIVERSIDAD AUTÓNOMA METROPOLITANA \\ CIUDAD DE MÉXICO, MÉXICO \\ https://orcid.org/0000-0002-1385-2398
}

\title{
RESUMEN
}

Las mujeres en México se han enfrentado a una serie de obstáculos y dificultades para incorporarse a los espacios de toma de decisiones, lo cual se evidenció en el proceso electoral 2017-2018 en donde la violencia política por razones de género visibilizó los entramados de un sistema político que atenta contra los derechos de las mujeres y de las diversidades sexo-genéricas. En este artículo retomamos el caso de las candidaturas trans o muxes, asi como las renuncias masivas de mujeres de las llamadas "manuelitas". Ambos casos nos permiten reflexionar sobre los retos y retrocesos en el avance sustantivo de las mujeres.

PALABRAS CLAVE: participación política, mujeres, avance sustantivo, acciones afirmativas, paridad y política.

* Doctoranda en Antropología Física por la Escuela Nacional de Antropología e Historia (ENAH) de México donde desarrolla el proyecto de investigación: Violencia política, cuerpo y salud en presidentas municipales de Oaxaca, México; Maestra en Antropología Social por la ENAH con mención en Género y Política; Licenciada en Intervención Educativa con mención en Interculturalidad por la Universidad Pedagógica Nacional (UPN).

** Candidata a doctora en Ciencias Antropológicas por la Universidad Autónoma Metropolitana (UAM) unidad Iztapalapa, donde desarrolla el proyecto: Ciudadanías Plurales: Mujeres indígenas y participación política en Ecuador y México; Maestra en Ciencias Sociales con mención en Género y Desarrollo por FLACSO sede Ecuador y Licenciada en Antropología Social por la UAM. 


\begin{abstract}
Dossier América Latina: género y política

\section{ABSTRACT}

Women in Mexico have faced a series of obstacles in entering the decision-making sphere. It was evidenced in the 2017-2018 electoral process where gender-based political violence made visible the frameworks of the political system which undermines the rights of women and sex-generic diversities. In this article we return to the case of trans or muxe candidates, as well as the massive resignations of the socalled "manuelitas". Both cases allow us to reflect on the challenges and setbacks in the substantive advancement of women.
\end{abstract}

KEYWORDS: political participation, women, substantive progress, affirmative actions, parity and politics.

\title{
Introducción
}

La participación política de las mujeres en México ha significado un entramado de conflictos, posibilidades y potencialidades. La entrada "masiva" de las mujeres en la arena pública ha evidenciado el profundo enraizamiento de prácticas sexistas y androcéntricas que la política tiene en este país. En el proceso electoral 2017-2018 pudimos observar que se incurrió en diversas simulaciones, que tenían como finalidad sofisticar las viejas prácticas para burlar las leyes concernientes al avance sustantivo de las mujeres en la política.

El objetivo de este texto consiste en mostrar y discutir los mecanismos utilizados por los partidos y actores políticos para simular el cumplimiento de las leyes electorales que tienen que ver con la participación política de las mujeres, con la intención de mantenerlas al margen y dejarlas fuera de los espacios de toma de decisiones. Poniendo en marcha una maquinaria interna y externa para conservar el statu quo político, en el que hombres y mujeres le son útiles a la estructura política vigente.

Para el desarrollo de dicha discusión hemos dividido el texto en tres apartados: I) El escenario general de la participación política de las mujeres en México, en el que realizamos un recuento sobre la incorporación de las mujeres en la política; II) Análisis del proceso electoral 2017-2018, donde visibilizamos los hechos de violencia política por razones de género que fueron perpetrados por distintos actores políticos; y finalmente, III) Reflexiones finales, en el cual establecemos algunas líneas para la discusión sobre el avance sustantivo de las mujeres en la política nacional mexicana.

Este artículo es resultado de un seguimiento exhaustivo a la violencia política por razones de género que se presentó en el proceso electoral 2017-2018. Para el análisis recopilamos información de medios de comunicación, acuerdos y resoluciones del Instituto Nacional Electoral (INE) y de Organismos Públicos Locales Electorales (OPLE), así como la revisión de distintas fuentes bibliográficas. Consideramos que una aproximación casuística nos permitirá 
mostrar las particularidades de la cultura política vigente en México y los retos actuales que enfrentan las mujeres políticas en el país.

La participación de las mujeres en la vida pública, en la esfera política y en los espacios de toma de decisiones no tiene que ver únicamente con el incremento numérico del que hemos sido testigos, sino con la incorporación de acciones y agendas comprometidas con los derechos de niñas y mujeres. La sola presencia de las mujeres en los cargos es insuficiente para transformar los escenarios de desigualdad estructural que viven las mujeres en México, por lo que en este momento coyuntural es necesario cuestionarnos ¿hacia dónde va la participación de las mujeres? Y ¿cómo podemos lograr la igualdad sustantiva?

\section{El escenario general de la participación política de las mujeres en México}

Las mujeres mexicanas fueron reconocidas como ciudadanas de manera tardía. Si bien estuvieron dentro de los diversos procesos históricos y sociales tales como las luchas por la independencia (1810), la revolución mexicana (1910) y otros tantos movimientos que tenían como finalidad la democratización del Estado nacional mexicano no fue hasta 1947 que se reconoció su derecho al voto en el ámbito municipal y hasta 1953 a nivel nacional (Tuñón, 2002).

El reconocimiento de las mujeres al sufragio fue un hito para la historia moderna de México, sin embargo, este reconocimiento fue producto de las luchas y demandas de los movimientos de mujeres de aquella época tanto en el contexto nacional como en el internacional. Es importante recordar, que en México se llevó a cabo el Primer Congreso Feminista de Yucatán (1916) en el cual se discutieron temáticas tales como los derechos de las mujeres a la educación, a votar y ser votadas, a participar políticamente, a la educación sexual, entre otros (Alejandre y Torres, 2016). No es casual que la primera mujer electa como diputada local fuera Elvia Carrillo Puerto en el Congreso Local de Yucatán (1923), suceso que obedeció a una coyuntura política particular del estado (Espinosa y Jaiven, 2012).

La recién adquirida ciudadanía puso en jaque a la sociedad mexicana, pues se argumentaba que las mujeres no sabrían tomar decisiones y que podrían ser fácilmente manipuladas por sus esposos o por el párroco (Espinosa y Jaiven, 2012). Además, las mujeres que aparecían en la escena política eran sancionadas públicamente a través de notas de prensa difundidas en los periódicos de circulación nacional orientadas a poner en duda su honorabilidad y capacidad.

A lo largo de las siguientes décadas un número reducido de mujeres comenzaron a ser electas para ocupar los cargos de presidentas municipales, diputadas locales y federales, senadoras y gobernadoras. Estas mujeres que transgredieron las normas sociales establecidas evidenciaron la subrepresentación de las mujeres en la política mexicana. Hecho que no había sido problematizado sino hasta la última década del siglo XX.
Los dilemas de la participación política de las mujeres en México. Retos y retrocesos ante el avance sustantivo de las mujeres

Mónica Rosado Toledo Lizeth Pérez Cárdenas 


\begin{tabular}{|c|c|c|c|c|}
\hline \multirow{8}{*}{$\begin{array}{r}\text { Dossier } \\
\text { América Latina: } \\
\text { género y política }\end{array}$} & \multirow{2}{*}{ Nombre } & & & \\
\hline & & Periodo & Estado & Cargo \\
\hline & Elvia Carrillo Puerto & 1923 & Yucatán & Diputada local \\
\hline & Rosa Torres & 1923 & Mérida, Yucatán & Regidora \\
\hline & Aurora Meza Andraca & 1938 & $\begin{array}{l}\text { Chilpancingo, } \\
\text { Guerrero }\end{array}$ & $\begin{array}{l}\text { Presidenta } \\
\text { Municipal }\end{array}$ \\
\hline & Aurora Jiménez de Palacios & 1952 & Baja California & Diputada federal \\
\hline & María Lavalle Urbina & 1964 & Campeche & Senadora \\
\hline & Alicia Arellano Tapia & 1964 & Campeche & Senadora \\
\hline $\begin{array}{r}\text { Cuadro 1. } \\
\text { Pioneras de la participación } \\
\text { política en México }\end{array}$ & Rosario Ibarra de Piedra & 1988 & Coahuila & $\begin{array}{l}\text { Candidata } \\
\text { a la presidencia } \\
\text { de la República }\end{array}$ \\
\hline
\end{tabular}

Elaboración propia con datos del portal oficial del Instituto Nacional de las Mujeres (INMUJERES).

A nivel internacional la normativa comenzó a presionar sobre el tema de la participación política de las mujeres. Su antecedente lo encontramos en la Convención sobre los Derechos Políticos de la Mujer (1954); posteriormente, en el Pacto Internacional de Derechos Civiles y Políticos (1976); así como, la Convención sobre la Eliminación de Todas las Formas de Discriminación contra la Mujer (CEDAW) (1979); de igual forma la Conferencia Mundial en El Cairo (1994) y Beijing (1995); y a nivel regional, la Convención Interamericana para Prevenir, Sancionar y Erradicar la Violencia contra la Mujer, también llamada Belem do Pará (1996) (Entrena, 2005).

La década de los noventa significó un momento de transición política, social, económica y cultural para Latinoamérica, y en particular para México, pues las temáticas relacionadas a la igualdad entre hombres y mujeres resurgieron en un momento de exigencias internacionales para impulsar el adelanto de las mujeres en todas las esferas de la vida, y el espacio político no fue la excepción. Particularmente, y después del proceso electoral de 1988, caracterizado por la falta de transparencia, el Congreso de la Unión expidió el Código Federal de Instituciones y Procedimientos Electorales (COFIPE), así como creó el Instituto Federal Electoral (IFE) ${ }^{1}$ (1990) como un órgano constitucional autónomo, que tenía entre sus funciones el organizar, legitimar y garantizar elecciones democráticas en nuestro país.

A partir de los cambios políticos y constitucionales ante una democracia emergente, se comenzaron a generar acciones encaminadas a promover la participación política de las mujeres, a través de políticas de género. Según Judith Astelarra (2004), tales políticas son el resultado de una serie de acciones

1 Con la reforma político-electoral de 2014 el Instituto Federal Electoral (IFE) pasaría a ser sustituido por el ahora Instituto Nacional Electoral (INE). 
y medidas que los Estados han promovido para transformar y eliminar las desigualdades que las diferencias de género han producido. Dichas acciones son producto de procesos históricos y las demandas de movimientos feministas y de mujeres.

En 1993, México incorporó una recomendación al COFIPE, la cual hacía énfasis en la promoción de la participación política de las mujeres. Sin embargo, no fue hasta 1996 que se aplicaron acciones afirmativas, las cuales para Fernández Poncela (1997) pueden definirse como aquellas acciones cuyo objetivo es borrar o hacer desaparecer la discriminación existente, a través de políticas concretas que sirven para igualar las oportunidades y vencer las resistencias, dificultades, obstáculos y limitaciones.

En esta primera etapa, se estableció en el COFIPE el umbral de $30 \%$ para mujeres y $70 \%$ para hombres en candidaturas titulares y suplentes. Posteriormente, en 2002, se estableció la cuota mínima para mujeres propietarias; y en 2008 se instruyó que las candidaturas deberían contar con “al menos el cuarenta por ciento de candidatos propietarios de un mismo género, procurando llegar a la paridad" (COFIPE 2008, artículo 219).

El logro de la incorporación de las mujeres a la política fue resultado de un camino arduo. Numerosos obstáculos han sido sorteados por las mujeres en la arena política, debido a la simulación en el cumplimiento de las normas electorales vigentes hasta ese momento. Consideramos pertinente traer a esta discusión lo sucedido en el proceso electoral federal de 2009, periodo correspondiente a elecciones intermedias en el cual se elegiría el total de la Cámara de Diputados. En aquella ocasión las mujeres mexicanas nos enteramos de que nueve diputadas federales electas por el principio de representación proporcional renunciaron al cargo, dejando a sus suplentes varones como titulares de dicho encargo. El caso fue conocido como "las Juanitas"2 y tuvo tal trascendencia que sentó las bases a través de una serie de sentencias emitidas por el Tribunal Electoral del Poder Judicial de la Federación (TEPJF), entre las que destaca la SUP-JDC-12624/2011 que tuvo como finalidad obligar a los partidos políticos a postular candidaturas propietarias y suplentes de un mismo género y de manera alternada sin importar el método de selección de candidaturas dentro de los partidos y coaliciones propuestas.

El movimiento amplio de mujeres, las luchas por la incorporación a los espacios de toma de decisiones, acuerdos y negociaciones para alianzas políticas entre ellas, acciones afirmativas, sentencias, resoluciones, reformas y normativas tuvieron como resultado el reconocimiento de la paridad como principio constitucional en 2014, como parte de la reforma político-electoral en ese mismo año.

2 El nombre "las Juanitas" responde al caso de Rafael Acosta Naranjo, alias Juanito, quién en las elecciones de 2009 se postuló como candidato a jefe delegacional de Iztapalapa por el Partido del Trabajo (PT) en el Distrito Federal, ahora Ciudad de México, con la condición de renunciar al cargo y cederlo a Clara Brugada, según un acuerdo de cúpulas partidarias.
Los dilemas de la participación política de las mujeres en México. Retos y retrocesos ante el avance sustantivo de las mujeres

Mónica Rosado Toledo Lizeth Pérez Cárdenas 
Dossier América Latina: género y política

\section{Cuadro 2.}

Acciones afirmativas para impulsar la participación política de mujeres en México

\begin{tabular}{lll}
\hline Año & \multicolumn{1}{c}{ Acción } & \multicolumn{1}{c}{ Porcentaje para mujeres } \\
\hline 1993 & Reforma al COFIPE $^{*}$ & Recomendación sin porcentaje \\
\hline 1996 & Reforma al COFIPE $^{*}$ & $\begin{array}{l}30 \% \text { en candidaturas titulares } \\
\text { o suplentes }\end{array}$ \\
\hline 2002 & Reforma al COFIPE & $30 \%$ en candidaturas propietarias \\
\hline 2008 & Adición al Código Federal de & $40 \%$ en candidaturas propietarias \\
& Procedimientos Electorales (COFIPE) & \\
\hline 2011 & Sentencia SUP-JDC-12624/2011 & $40 \%$ de candidaturas propietarias \\
& & y suplentes del mismo género \\
\hline 2014 & $\begin{array}{l}\text { Reforma político-electoral. Constitución } \\
\text { Política de los Estados Unidos Mexicanos. }\end{array}$ & $\begin{array}{l}50 \% \text { de candidaturas propietarias } \\
\text { Art. 41 }\end{array}$ \\
\hline
\end{tabular}

*Estas reformas carecían de obligatoriedad.

Elaboración propia a partir de Medina (2010) y portal oficial del Instituto Nacional Electoral (INE).

Las elecciones intermedias que se llevaron a cabo en 2015 fueron el primer ejercicio en el que la paridad se puso en marcha. Los resultados de esta elección según el Instituto Nacional Electoral (INE) en la Cámara de Diputados representaron el 57,4 \% para hombres y el 42,6 \% para mujeres. Estos porcentajes reflejan un avance numérico considerable en los espacios legislativos federales y dan cuenta de un empuje constante hacia la paridad en estos órganos legislativos. Por lo tanto, consideramos importante mostrar un panorama general de los sucesos en el proceso electoral 2017-2018.

\section{Análisis del proceso electoral 2017-2018}

El proceso electoral 2017-2018 es considerado el más grande de la historia democrática de nuestro país. Según datos del INE, en éste se disputaron un total de 3400 cargos locales y federales, entre los que encontramos: presidencia de la república, diputaciones federales, senadurías, gubernaturas, diputaciones locales, así como presidencias y alcaldías municipales. La característica de este proceso estuvo acompañada por la paridad, lo que significó que por lo menos la mitad de las candidaturas fueron disputadas por mujeres.

En este escenario de la contienda electoral, un fenómeno se hizo presente, la crisis de violencia e inseguridad generalizada en el territorio nacional. Hecho que tuvo como consecuencia que el proceso electoral fuera señalado como el más violento de la historia contemporánea de México. Por ejemplo, el Sexto Informe de Violencia Política en México 2018, de la Unidad de Análisis en Riesgos de Seguridad de Etellekt Consultores señala que se presentaron 581 
agresiones globales en contra de políticos en el país, de las cuales “ 136 fueron asesinatos, 197 políticos amenazados y/o intimidados, 70 agresiones físicas, 52 agresiones con armas de fuego (tentativa de homicidio), 51 atentados contra familiares de políticos, 36 asaltos con violencia, 20 secuestros e intentos de secuestro y 19 heridos por armas de fuego" (Etellekt, 2018, p. 4), mostrando un panorama desolador.

Primero, es importante mencionar que la violencia política estuvo presente en el proceso electoral y resulta fundamental señalar que dicha violencia afectó a cuerpos concretos de formas diferenciadas. En ese sentido, consideramos un elemento clave para comprender el proceso electoral 2017-2018, la violencia política por razones de género que vivieron candidatas, activistas y mujeres en el escenario político. Al respecto, se puede decir que la violencia política por razones de género es una problemática de reciente data, y no porque antes no se presentara, sino porque se había comprendido y conceptualizado de una manera distinta.

Hace algunas décadas, diversos estudios (Barrera y Massolo, 1998; Barrera y Massolo, 2003; Barrera y Suárez, 2012) hablaban de los obstáculos y costos que entrar al mundo de la política representaba para las mujeres, sin embargo, a partir de una serie de hechos sistemáticos que se perpetraron contra las mujeres fue necesario señalar con claridad y conceptualizar el fenómeno, el cual fue definido inicialmente por la Asociación de Concejalas de Bolivia (ACO$\mathrm{BOL})$ quienes definieron este tipo de violencia como:

Las acciones y/o conductas agresivas cometidas por una persona, por sí o a través de terceros, que causen daño físico, psicológico o sexual en contra de una mujer y/o su familia, en ejercicio de la representación política, para impedir y restringir el ejercicio de su cargo o inducirla a tomar decisiones en contra de su voluntad, de sus principios y de la ley. (ACOBOL 2007)

Posteriormente, el concepto comenzó a ser retomado, debatido y discutido, generando profundas reflexiones con la finalidad de comprender el escenario al que se enfrentaban las mujeres en la esfera política. De tal suerte que Mona Lena Krook y Juliana Restrepo señalan que "[l]a violencia y el acoso político contra las mujeres describe comportamientos dirigidos específicamente contra las mujeres por ser mujeres con el propósito de que abandonen la política, presionándolas para que renuncian a ser candidatas o a un cargo político en particular" (Krook y Restrepo, 2016, p. 130). Es decir, la violencia política contra las mujeres tiene la intención de que las mujeres se retiren o desistan de participar en el escenario político, y este tipo de violencia es accionada por diversos actores políticos, así como otros actores sociales presentes en los territorios.

El proceso electoral 2017-2018 nos mostró diferentes expresiones de violencia política por razones de género contra las mujeres. Las mujeres que participaron, contendieron y lograron llegar a la ocupación del cargo recorrieron
Los dilemas de la participación política de las mujeres en México. Retos y retrocesos ante el avance sustantivo de las mujeres

Mónica Rosado Toledo Lizeth Pérez Cárdenas 
Dossier América Latina: género y política un largo camino, enfrentando una serie de acciones y estrategias por parte de partidos políticos y medios de comunicación que vulneraron sus derechos. El escenario político se caracterizó por formas diversas de violencia política y las mujeres fueron blanco particular de agresiones a través de notas de prensa, rumores difundidos en medios de comunicación, y en general, descalificaciones por el simple hecho de ser mujeres. Particularmente hubo dos situaciones que consideramos necesario discutir y problematizar.

\section{La diversidad, los muxes y la violencia política}

El estado de Oaxaca tiene el mayor número de municipios del país: 570, lo cual representa todo un reto para llevar a cabo las elecciones en las 8 regiones diversas en lo geográfico, social, cultural y político; tiene dos sistemas de elección de representantes reconocidos por la Constitución Política del Estado, sistema de Partidos Políticos (153 municipios) y Sistemas Normativos Indígenas (SNI) -anteriormente llamados de Usos y Costumbres (UyC)- (417 municipios). Lo anterior muestra los diversos matices y las diferentes maneras de entender y hacer política en lo local de los municipios clasificados como de mayoría indígena que integran el estado de Oaxaca, el segundo con mayor diversidad étnica del país. En este escenario tan particular, el proceso electoral 2017-2018 tuvo, como en todo el país, una participación ciudadana sin precedentes, pues además de la elección de cargos federales se renovarían 153 presidencias municipales y la totalidad del Congreso Local.

Por causa de la participación cada vez mayor de mujeres, candidaturas independientes, candidaturas de personas transgénero, transexuales, intersexuales $o$ muxes $^{3}$ y en algunos casos la simulación de serlo para burlar la ley e inscribirse como parte de las cuotas de género, se hicieron presentes distintos conflictos y violencia política, los cuales ponen en evidencia la resistencia a los procesos democráticos y la respuesta de las autoridades electorales al respecto.

El Instituto Estatal Electoral y de Participación Ciudadana de Oaxaca (IEEPCO) aprobó Lineamientos en materia de paridad de género ${ }^{4}$ y derivado del artículo 16, contempló la obligatoriedad de los partidos políticos a cumplir con la postulación de candidaturas paritarias, y que las personas transgénero, transexuales o muxe pudieran contender con el género que eligieran y se auto adscribieran. A esta convocatoria se inscribieron con el nombre que les

3 Hombres indígenas zapotecas del Istmo de Tehuantepec, que tienen preferencias sexuales por otros hombres sin considerarse homosexuales, pues se consideran mujeres atrapadas en cuerpos de hombres, pero sin necesariamente ser transexuales (algunos se visten como mujeres y otros no). Para una definición más amplia consulte Miano, Marinella (2002), Hombre, mujer y muxe en el Istmo de Tehuantepec, Ed. Plaza y Valdés. INAH.

4 Consultado en: http://www.ieepco.org.mx/archivos/acuerdos/2017/ANEXO \%20LINEAMIENTOS.pdf 
fue asignado 19 personas que decían ser parte de la comunidad transexual o muxe, originarios de las diversas regiones y municipios del estado.

Todas las candidaturas fueron aprobadas por el Consejo General del IEEPCO el 20 de abril de 2018 (IEEPCO, Acuerdo IEEPCO-CG-32/2018), el cual emitió una lista con los datos con el objetivo de difundir la acción afirmativa puesta en marcha en ese periodo electoral. La instrucción del IEEPCO para fomentar la inclusión de diversos grupos de la sociedad civil, en este caso los transexuales o muxes, fue utilizada por algunos actores y partidos políticos de manera fraudulenta para ocupar espacios que correspondían a las mujeres dentro de la normatividad hacia una postulación paritaria, tomando ventaja de un vacío legal.

Lo que no contemplaron fue que, después de haber realizado una investigación en redes sociales para determinar si las 19 personas postuladas pertenecían al género que decían ser, las asociaciones civiles Ladxidó Muxe, Auténticas Intrépidas Buscadoras del Peligro, el Colectivo Binni Laanu A.C y otros activistas muxes, entre quienes se encontraba Amaranta Gómez Regalado ${ }^{6}$, fueron quienes denunciaron formalmente el intento de los partidos para postular a hombres heterosexuales simulando ser mujeres trans o muxes. Ante los hechos suscitados, el IEEPCO inició una investigación a través de un Procedimiento Ordinario Sancionador para dar atención a la denuncia (IEEPCO, Expediente CQDPCE/POS/005/2018) y la Comisión de Quejas y Denuncias del IEEPCO empezó las investigaciones correspondientes que, de hallar una falta a la norma, concluiría en sanciones que irían desde una multa al partido, hasta la cancelación de los registros de dichas candidaturas.

En un comunicado de prensa emitido el 1 de junio de 2018, el Consejo General del IEEPCO canceló definitivamente 17 candidaturas presentadas como trans "al determinar que se violaron principios constitucionales y leyes generales, así como locales en materia de paridad de género por parte de las personas, coaliciones y partidos denunciados". Lo anterior fue notificado tanto a los partidos políticos como a las personas denunciadas.

De acuerdo con el documento "Los derechos políticos de las personas trans: El caso Oaxaca en las elecciones de 2018", de las 17 notificaciones se obtuvo respuesta de 13 candidatos y de 4 partidos políticos, Partido Acción Nacional (PAN), Partido de la Revolución Democrática (PRD), Movimiento Ciudadano (MC) y Partido Revolucionario Institucional (PRI), quienes argumentaron entre otros motivos: que en los lineamientos no se establecía que tuvieran que acreditar su identidad de género o que tuvieran que cumplir requisitos adicionales, puesto que bastaba autoadscribirse como tal para registrarse, que desconocían el contenido del documento presentado ante el IEEPCO para

5 Por una cuestión administrativa, a manera de comprobar su identidad como ciudadanos mexicanos con la credencial del Instituto Nacional Electoral (INE), entre otros documentos.

6 En 2003, contendió por primera vez con su identidad de género, es decir como muxe, en el municipio de Juchitán Oaxaca.
Los dilemas de la participación política de las mujeres en México. Retos y retrocesos ante el avance sustantivo de las mujeres

Mónica Rosado Toledo Lizeth Pérez Cárdenas 
Dossier América Latina: género y política su registro como candidata trans y que se enteraron a través de los medios de comunicación o por redes sociales. Las respuestas por parte de los partidos no fueron muy distintas, pues argumentaron que colaboradores al interior de algunos partidos habrían inscrito sin consentimiento a varios aspirantes a manera de perjudicarlos, y sólo cuatro candidatos presentaron su renuncia.

Tanto el IEEPCO como la Sala Superior del Tribunal Electoral del Poder Judicial de la Federación (TEPJF) evaluaron y acordaron la mejor manera de reconocer los derechos políticos de las personas trans y al mismo tiempo evitar en futuras contiendas los fraudes y simulaciones a la ley de paridad en las candidaturas, por lo que hicieron énfasis en que las 17 personas realizaron fraude a la ley con la clara intención de apropiarse de los lugares que por ley les correspondían a las mujeres. Se determinó que los candidatos que intentaron hacer fraude a la ley no podrían participar en la contienda, (IEEPCO, Acuerdo IEEPCO-CG-45/2018). Sin embargo, el 22 de junio de 2018, la Sala Superior del TEPJF revocó el acuerdo emitido por el IEEPCO y por mayoría de votos confirmó dos de las 15 candidaturas transgénero postuladas en Oaxaca. Además, resolvió que la autoadscripción a un género se dé a partir del reconocimiento de la comunidad y que las autoridades electorales se encarguen de vigilar el buen uso de esta medida afirmativa.

Finalmente, el IEEPCO recalcó el buen uso de los lineamientos en materia de paridad de género para las candidaturas de Kristel Ramírez Cortés, candidata a la Presidencia Municipal de San Pedro Mixtepec, Felina Santiago Valdivieso y Mística Sánchez Gómez, propietaria y suplente a diputadas locales en el distrito de Juchitán de Zaragoza, quienes cumplieron con los requerimientos y acreditaron su identidad de género. Las candidaturas trans o muxes fraudulentas o simuladas violentan los derechos políticos de las personas que forman parte de la diversidad sexo-genérica como de los derechos políticos de las mujeres, por lo que es necesario convocar a los partidos políticos a respetar las leyes electorales y hacer valer los derechos de todas las personas.

\section{Renuncias masivas de mujeres, las Ilamadas "manuelitas"7}

La jornada electoral del 1 de julio de 2018 tuvo importantes avances. Las mujeres obtuvieron 241 diputaciones, lo que representa el 48,2 \%, así como 63 senadurías que representan el 49,2\%. Estos números significaron un adelanto significativo para las mujeres en política, ya que fue la primera vez que se alcanzó la paridad numérica en los espacios legislativos más importantes de

7 Se les llamó “manuelitas” haciendo alusión al nombre del gobernador de Chiapas Manuel Velasco quién pidió licencia para contender por una senaduría. Para ampliar información consultar la Opinión: Paridad de género y violencia política: las “Manuelitas de Chiapas” en: https:// www.eluniversal.com.mx/observatorio-nacional-ciudadano/paridad-de-genero-y-violenciapolitica-las-manuelitas-de-chiapas 
nuestro país. Ante dichos resultados parecía que el avance de las mujeres no se podía detener. Sin embargo, lo que sucedió posteriormente a nivel local dio muestra de una serie de estrategias articuladas por los partidos políticos para burlar la norma y de esta forma anular y revertir la incorporación de las mujeres en espacios de toma de decisiones.

A lo largo del territorio nacional las mujeres ocuparon un porcentaje importante tanto en los congresos locales como en los gobiernos municipales, sin embargo, el 7 de septiembre un grupo de mujeres presentaron sus renuncias como regidoras y diputadas en el estado de Chiapas. Hecho que rápidamente fue visibilizado por los medios de comunicación y discutido por el Instituto Electoral del Estado de Chiapas (IEPC) así como por el Instituto Nacional Electoral (INE), ya que la situación evidenciaba una manipulación de las normas electorales para poder incumplir el principio de paridad de género, así como una sofisticación de actos ilícitos de diferentes partidos para violentar los derechos políticos de las mujeres.

Ante este escenario un grupo de diputadas federales de distintas filiaciones políticas hicieron un llamado al gobernador del estado de Chiapas Manuel Velazco, ante el pleno de la Cámara de Diputados, para terminar con la violencia política recibida por las 43 mujeres electas para regidoras y legisladoras ${ }^{8}$. Aunado a ello el 12 de septiembre de 2018 el IEPC emitió el acuerdo: IEPC/ CG-A/179/2018 "Acuerdo del Consejo General del Instituto de Elecciones y Participación Ciudadana por el que se realiza la asignación de diputaciones por el principio de representación proporcional a los partidos políticos con derecho a ello, en el marco del proceso electoral local ordinario 2017-2018" y, al mismo tiempo, el INE expidió la resolución INE/CG1307/2018 "Resolución del Consejo General del Instituto Nacional Electoral, por el que se ejerce facultad de atracción y se emiten criterios de interpretación para la asignación de diputaciones e integrantes de los ayuntamientos, en relación con el principio de paridad de género" ${ }^{\prime \prime}$.

Más allá de las notas de prensa que pudieron ser recuperadas sobre las renuncias masivas, la resolución del INE daba cuenta que al día 10 de septiembre de 2018 se habían presentado un total de 66 renuncias ratificadas, encontrándose vigentes 41 para diputaciones locales (27 mujeres y 14 hombres) y 25 para ayuntamientos (24 mujeres y 1 hombre). Asimismo, posterior a la elección, 43 mujeres renunciaron a sus candidaturas y en el caso del Partido Verde Ecologista de México (PVEM) renunciaron todas las candidatas postuladas a una diputación de representación proporcional, hecho que

8 Para mayor información consultar: Ni "manuelitas” ni “juanitas”: condenan en San Lázaro violencia electoral de género en Chiapas en https:/www.proceso.com.mx/550554/ni-manuelitas-ni-juanitas-condenan-en-san-lazaro-violencia-electoral-de-genero-en-chiapas

9 Consultado en: http://www.iepc-chiapas.org.mx/archivos/sesiones/acuerdos/2018/IEPC CG_A_179_2018.pdf

10 Consultado en: https://repositoriodocumental.ine.mx/xmlui/bitstream/handle/12345 6789/98353/CGex201809-12-rp-unico.pdf
Los dilemas de la participación política de las mujeres en México. Retos y retrocesos ante el avance sustantivo de las mujeres

Mónica Rosado Toledo Lizeth Pérez Cárdenas 
Dossier América Latina: género y política evidenció la profunda misoginia de dicho partido político al intentar ocupar esos espacios por varones. Ante estos sucesos el INE en uso de sus facultades señaló que en caso de renuncia o cancelación por parte de una lista esta tendrá que ser asignada a la que sigue respetando invariablemente el principio de género, por lo que en ninguna circunstancia se le asignaría a una de un género distinto y en caso de hacerlo de esa manera se estarían infringiendo una serie de principios legales.

La resolución tiene 3 elementos que nos parece muy importante destacar: 1) las diputaciones asignadas por representación proporcional ${ }^{11} y$ que corresponden a mujeres serán para mujeres, lo que significa que en caso de que en un partido político las fórmulas hubieran renunciado entonces la diputación será asignada a mujeres que no hubiesen obtenido el triunfo por el principio de mayoría relativa ${ }^{12}$ y contarán con el mayor porcentaje de votos; 2) en el caso de las regidurías de representación proporcional, al no haber mujeres por renuncia o cancelación, las regidurías serán asignadas a las fórmulas integradas por mujeres y que hayan contenido por el principio de mayoría relativa respetando el orden de prelación; y 3) si no se pudieran cubrir los cargos de diputaciones y regidurías de representación proporcional dentro del mismo partido, deberán ser reasignadas entre los otros partidos a las fórmulas conformadas por mujeres.

Tanto el INE como el IEPC mostraron compromiso, voluntad política e imparcialidad en la emisión de resoluciones y acuerdos en los casos de violencia política de género presentados. Queremos señalar que a la fecha se desconoce las condiciones en que las mujeres se encuentran ejerciendo sus cargos, debido a que por un lado está la norma y por otro lado están las condiciones estructurales y una cultura política misógina que atenta contra los derechos políticos de las mujeres al dificultar el acceso y permanencia de las mujeres en la política.

\section{Reflexiones finales}

Desde que las mujeres mexicanas obtuvieron el derecho al voto, han incursionado paulatinamente en la vida política del país. Las diferentes luchas feministas por el reconocimiento de los derechos de las mujeres, así como la implementación de medidas legislativas en México han tenido como resultado que en la actualidad nuestro país se coloque como un referente en materia de

11 "Principio de elección basado en la asignación de cargos de representación popular tomando como base el porcentaje de votos obtenidos por un partido político en una región geográfica" Sistema de Información Legislativa (SIL), consultado en: http://sil.gobernacion.gob.mx/ Glosario/definicionpop.php?ID=210

12 “Tipo de votación que tiene por principio elegir a quien tenga el mayor número de votos emitidos” Sistema de Información Legislativa (SIL), consultado en http://sil.gobernacion.gob. $\mathrm{mx} /$ Glosario/definicionpop.php?ID=153 
paridad política para la región, sin embargo, el proceso ha estado acompañado de numerosos obstáculos que se articulan y reconfiguran con la finalidad de sostener el sistema político mexicano.

Las mujeres en política se han enfrentado a diversas expresiones de violencia política por razones de género y constantemente tienen que hacer frente a acciones que vulneran su integridad y su capacidad tanto en lo personal como en lo político. Continuamente son agredidas e intimidadas antes, durante y después del proceso electoral, por lo que para ellas la violencia no cesa. Aunada a la violencia específica contra algunas mujeres en particular, existe una violencia sistemática dirigida a las mujeres como grupo y que generalmente es orquestada desde las cúpulas de los partidos políticos.

Los dos casos a los que hicimos referencia develan la constante resistencia de los partidos políticos a acatar la norma, además de obedecer a un sistema machista y misógino que coloca a las mujeres y a las diversidades sexo-genéricas en posiciones de desventaja. Por lo tanto, sostenemos que los partidos políticos en México se han aprovechado de los vacíos legales, al mismo tiempo que han manipulado la normatividad vigente de tal suerte que les favorezca, pero sobre todo beneficie los intereses de los grupos de poder mayoritarios.

Consideramos que el pasado proceso electoral nos dejó lecciones importantes, por un lado, una incorporación masiva de mujeres a los espacios de toma de decisiones en los tres niveles de gobierno, ya que por primera vez contamos con un gabinete y congreso paritarios, lo que demuestra una aplicación conforme a derecho de las normas electorales; y por otro lado, las estrategias de simulación para excluir a las mujeres y a las diversidades sexo-genéricas, lo cual de alguna manera fue subsanado y sancionado por las autoridades responsables, reduciendo el perjuicio al adelanto sustantivo de las mujeres.

\section{Referencias bibliográficas}

Alejandre, G. L. y Torres E. (2016). El Primer Congreso Feminista de Yucatán 1916. El camino a la legislación del sufragio y reconocimiento de ciudadanía a las mujeres. Construcción y tropiezos. Revista de Estudios Políticos y Sociales, vol. 39 (septiembrediciembre), pp. 59-89.

Asociación de Concejalas de Bolivia (ACOBOL) (1994). Guía de información y prevención contra el acoso político y la violencia de género en razón de género. Quito: REPEM.

Barrera, D. y Massolo, A. (Coords.) (1998). Mujeres que gobiernan municipios. Experiencias, aportes y retos. México: Colegio de México, Programa Interdisciplinario de Estudios de la Mujer.

Barrera, D. (2004). Transgresiones, control social y violencia hacia las mujeres en el ámbito rural. GénEros. Universidad de Colima, Centro Universitario de Estudios de Género, no. 31, octubre, año 11, pp. 54-60.
Los dilemas de la participación política de las mujeres en México. Retos y retrocesos ante el avance sustantivo de las mujeres

Mónica Rosado Toledo Lizeth Pérez Cárdenas 
Dossier América Latina: género y política
Barrera, D. (2007). Equidad de género y participación de las mujeres en los cargos de elección popular a 54 años del voto femenino en México. Sororidad, Revista Trimestral del Instituto Veracruzano de las Mujeres, octubre/noviembre, pp. 9-16.

Barrera, D. y Suárez, B. (2012). San Román, Los desafíos de llegar y de ejercer un cargo. Mujeres mexicanas en los albores del siglo XXI. Revista Ra Ximhai, vol. 8, no. 1 enero-abril. El Fuerte: Universidad Autónoma Indígena de México.

Código Federal de Instituciones y Procedimientos Electorales (2008). Publicado el 14 de enero de 2008. Recuperado de: http://ieepco.org.mx/biblioteca_digital/legislacion/ COFIPE.pdf

Entrena, L. (2005). Constitución y acciones positivas. El sistema de cuotas de participación política para mujeres en Ecuador. Anuario de Derecho Constitucional Latinoamericano. Recuperado 19.03.2019 de https://revistas-colaboracion.juridicas.unam.mx/index.php/ anuario-derecho-constitucional/article/viewFile/30269/27323

Espinosa, D., Lau Jaiven, A. (Coords.) (2011). Un fantasma recorre el siglo: luchas feministas en México 1910-2010. México, D.F: Universidad Autónoma Metropolitana. Unidad Xochimilco, Editorial Itaca.

IEEPCO (2018). Los derechos politicos de las personas trans. El caso Oaxaca en las elecciones de 2018. Recuperado de http://www.ieepco.org.mx/archivos/documentos/2018/art \%C3 \%ADculos \%20de \%20inter \%C3 \%A9s/Los \%20derechos \%20poli \%CC \%81ticos \%20de \%20las \%20personas \%20transexuales..pdf

Miano, M. (2002). Hombre, mujer y muxe en el Istmo de Tehuantepec. México: Plaza y Valdés, Escuela Nacional de Antropología e Historia (ENAH).

Centro de Estudios para el Adelanto de las Mujeres y la Equidad de Género (CEAMEG) (2010). La participación política de las mujeres. De las cuotas de género a la paridad. México: Cámara de Diputados LXI Legislatura.

Tuñón, E. (2002) ¡Por fin! ya podemos elegir y ser electas. México: Ed. Plaza y Valdés, INAH. 